\title{
FIBROSIS QUÍSTICA ATÍPICA Y ENFERMEDAD DEL CFTR: A PROPÓSITO DE UN CASO DE TRAQUEOBRONCOPATÍA OSTEOCONDROPLÁSTICA
}

\author{
ATYPICAL CYSTIC FIBROSIS AND CF RELATED DISEASE. A CASE OF \\ OSTEOCHONDROPLASTIC TRACHEOBRONCHOPATHY
}

Dra. María G. Parra 0. 1,2, Dr. Rodrigo I. Bozzo H. 1, Dra. María A. Palomino M. 1,3

1. Pediatra Especialista en Enfermedades Respiratorias, Equipo Fibrosis Quística Hospital Roberto del Río

2. Pediatra Especialista en Enfermedades Respiratorias. Jefe Equipo Fibrosis Quística

3. Pediatra Especialista en Enfermedades Respiratorias. Profesor Asociado. Departamento de Pediatría Norte, Universidad de Chile

\begin{abstract}
ATYPICAL CYSTIC FIBROSIS AND CF RELATED DISEASE. A CASE OF OSTEOCHONDROPLASTIC TRACHEOBRONCHOPATHY
\end{abstract}

Cystic fibrosis (CF) is a genetic common disease within the white population, caused by mutations in the CF transmembrane conductance regulator gene (CFTR). It mainly involves progressive respiratory diseases and pancreatic exocrine insufficiency. Atypical CF represents approximately $2 \%$ of cases and affects adolescents or adults with pancreatic exocrine sufficiency, normal or borderline sweat chloride test, or presenting a single clinical feature, such as chronic rhinosinusitis, nasal polyposis, pancreatitis, biliary cirrhosis, portal hypertension, or obstructive azoospermia. Clinical heterogeneity depends on the amount of functional protein, which is influenced by the type of mutation. Other genes and environmental exposure could modify the phenotype. Certain conditions may result from CFTR dysfunction without fulfilling diagnostic criteria for CF: the term CFTR-related disease is used to describe a single organ disease, and in cases where a mild CF phenotype is apparent, it is called atypical CF. We describe a case of osteocondroplastic tracheobronchopaty as a form of mild presentation of atypical CF or CFTR-related disease.

Keywords: cystic fibrosis, cystic fibrosis transmembrane conductance regulator, pancreatitis, azoospermia, sinusitis, osteochondroplastic tracheobronchopathy

\section{RESUMEN}

La fibrosis quística (FQ), enfermedad genética frecuente de la raza blanca, es causada por la mutación del gen que codifica para la proteína reguladora de transmembrana (CFTR). Produce principalmente una enfermedad respiratoria progresiva e insuficiencia pancreática. La FQ atípica representa el 2\% de los casos. Aparece en adolescentes y adultos con suficiencia pancreática, test del sudor normal o dudoso o solo un sistema afectado: sinusitis crónica, poliposis nasal, pancreatitis, cirrosis biliar, hipertensión portal o azospermia obstructiva. La heterogeneidad clínica depende de la cantidad de CFTR funcionante, la que está influenciada por el tipo de mutación. Otros genes o la exposición ambiental podrían modificar el fenotipo. Cuando existe un órgano comprometido se la ha llamado enfermedad relacionada CFTR; en casos leves es el término FQ atípica. Esta revisión describe un paciente con traqueobroncopatía osteocondroplástica como forma de presentación de FQ atípica o de enfermedad relacionada a CFTR.

Palabras clave: fibrosis quística, regulador de transmembrana, sinusitis, pancreatitis, azoospermia, traqueobroncopatía osteocondroplástica

\section{INTRODUCCIÓN}

La fibrosis quística es la enfermedad genética recesiva más frecuente de la raza blanca, es causada por la mutación del gen que codifica para la proteína reguladora de transmembrana - cystic fibrosis transmembrane conductance regulator, CFTRla que está compuesta por 1.480 aminoácidos y se localiza en la membrana de las células epiteliales. La proteína CFTR

\section{Correspondencia:}

Dra María Angélica Palomino M

Hospital Roberto del Río

Zañartu 1085 - Independencia

Santiago, Chile

Correo electrónico: mapalomino@me.com regula el flujo de electrolitos y agua; su disfunción ocasiona una alteración del transporte de cloro y sodio, produciendo un moco espeso y viscoso que obstruye los conductos del órgano en el cual se localiza, produciendo principalmente enfermedad respiratoria progresiva e insuficiencia pancreática $(1,2)$. El diagnóstico de $F Q$ exige un cuadro clínico característico y un test del sudor (TS) sobre $60 \mathrm{mEq} / \mathrm{l}$, con la identificación de dos mutaciones conocidas como causantes de esta enfermedad idealmente (1-5). La mutación más frecuente causa la pérdida del aminoácido fenilalanina en la posición 508 de la proteína CFTR (F508del), la cual está presente en el 75\% de los alelos de FQ; aproximadamente el $40 \%$ de los pacientes con FQ son heterocigotos para esta mutación $(1,2)$ y en Chile la prevalencia alcanza al 30\% (4). En la FQ clásica los síntomas pueden estar 
presentes desde el nacimiento o aparecer en el tiempo con enfermedad respiratoria crónica, gastrointestinal, insuficiencia pancreática en el $90 \%$ de los casos e infertilidad masculina (16). En el $2 \%$ de los casos la FQ se presenta de forma atípica, siendo a menudo menos sintomática durante la niñez, por lo cual se diagnostica en general en la adolescencia y adultez $(7,8)$. Estos pacientes se caracterizan por presentar un compromiso pulmonar leve y suficiencia pancreática exocrina, a menudo con un solo órgano o sistema afectado, un TS normal o poco elevado, la detección de dos mutaciones del gen CFTR, siendo una de ellas leve y/o el potencial nasal alterado $(7,8)$.

\section{CASO CLÍNICO}

En esta revisión describimos un caso clínico de una FQ atípica controlado en el Hospital Roberto del Río, Santiago de Chile, destacando sus dificultades diagnósticas y aproximaciones terapéuticas. El niño fue sano hasta los 10 años y consultó por presentar tos persistente, sinusitis e intolerancia progresiva al ejercicio los dos años previos, con una pobre respuesta a tratamientos antibióticos y broncodilatadores, por lo cual fue derivado a nuestro centro desde el norte del país. Al ingreso el paciente estaba enflaquecido, con crepitaciones gruesas, sibilancias inspiratorias y espiratorias, sinusitis costrosa, atrofia de mucosa nasal y senos paranasales velados en la tomografía computada (TC). Se pesquisó Pseudomona aeruginosa en cultivos nasales y bronquiales, por esta razón se solicitaron varios TS, con cloro de 24, 25 y 24,8 mEq/l, además de cuantificación de inmunoglobulinas que resultó normal. La espirometría mostró una limitación obstructiva al flujo aéreo sin cambios post broncodilatador. La forma de la curva flujo-volumen fue sugerente de una obstrucción de vía aérea central y una disminución de flujos espiratorios máximos a volúmenes pulmonares altos (Figura 1). Debido a estos hallazgos se realizó una fibrobroncoscopía que mostró una traqueobroncopatía osteocondroplástica (Figura 2). La TC de tórax mostró bronquiectasias cilíndricas en lóbulos superior e inferior derechos y la biopsia de cilios fue normal, al igual que la elastasa-1 fecal. El estudio genético para $F Q$ con

Figura 1. Curva flujo / volumen

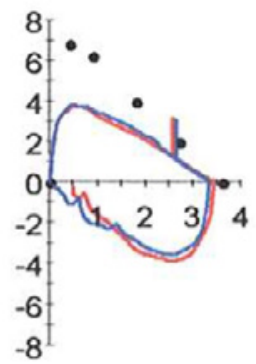

Paciente de 13 años con traqueobroncopatía osteocondroplástica secundaria a una fibrosis quística atípica o enfermedad asociada al CFTR. La forma de la curva orienta a una obstrucción fija de la tráquea, observándose disminución de los flujos espiratorios máximos a volúmenes pulmonares altos. La CVF fue de 3,43 L (Knudson 95\%, z score 0,01 GLI), el VEF1 2,58 L (Knudson 83\%, z score -1 GLI), FEF 2,24 $\mathrm{L}$ (Knudson 64\%, z score -1,6 GLI).

\begin{tabular}{lllll}
\hline$\bullet$ & Teórico $\longrightarrow$ & Pre & & \\
\hline
\end{tabular}

GLI: Global Lung Initiative

Figura 2. Visión fibrobroncoscópica

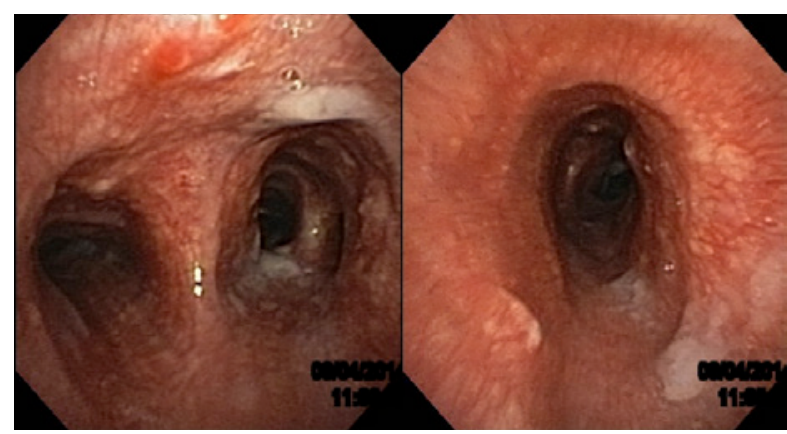

Traqueobroncopatía osteocondroplástica: se observan protuberancias en la mucosa de la pared anterior y lateral de la tráquea con aspecto de empedrado.
Figura 3. Curva Flujo / Volumen de control

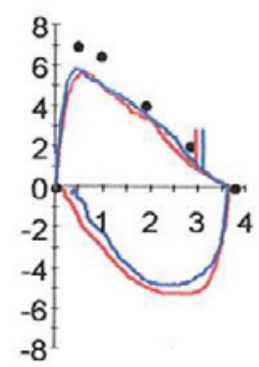

Curva flujo-volumen a los 15 años de edad, donde se observa mejoría parcial de la espirometría posterior al tratamiento con antibióticos endovenosos, kinesioterapia y alfa dornasa. La CVF fue de 3,65 L (Knudson 97\%, z score 0,29 GLI), VEF1 2,99 L (Knudson 93\%, z score -0,05 GLI), FEF 2,94 L (Knudson 64\%, z scoreGLI -1)

GLl: Global Lung Initiative 
33 mutaciones resultó ser heterocigoto para F508del, no siendo posible descartar $F Q$ con un estudio genético extendido o con potenciales nasales para obtener una evaluación funcional de la proteína CFTR. Se trató de acuerdo a protocolo de FQ con antibióticos endovenoso, alfa dornasa, tobramicina inhalada y azitromicina. La Pseudomona aeruginosa desapareció de los cultivos traqueales, mejoró la condición clínica, la tolerancia al ejercicio, su nutrición, la función pulmonar (Figura3), curva flujo volumen y el aspecto morfológico en la fibrobroncoscopía.

\section{TRAQUEOBRONCOPATÍA OSTEOCONDROPLÁSTICA}

Es una enfermedad rara, benigna, de evolución lenta y de diagnóstico generalmente entre la cuarta y sexta década de la vida. Se caracteriza por el hallazgo de nódulos osteocartilaginosos característicos de 1 a $6 \mathrm{~mm}$ que se observan en la submucosa traqueo bronquial, con aspecto de jardín de rocas o empedrado, que respetan la pared membranosa posterior de la tráquea y grandes bronquios y no guardan relación con los cartílagos traqueales normales, por esta razón no es requisito realizar biopsia para su diagnóstico (9-11). Existe además una atrofia de las glándulas submucosas y en ocasiones metaplasia escamosa e interposición de tejido de médula ósea (10). Algunos autores, basados en estudios de series de casos describen 3 fases: leucoplásica, cartilaginosa y ósea (9-13). Podría confundirse desde el punto de vista endoscópico con amiloidosis, sarcoidosis endobronquial, lesiones tuberculosas, papilomatosis y tumores. En general este proceso suele descubrirse en forma accidental en la edad adulta, ya sea como hallazgo endoscópico, durante procedimientos anestésicos o en una necropsia, porque puede ser asintomático (10). En ocasiones suele presentarse con tos seca persistente, disnea, odinofagia, disfonía, expectoración hemática o infecciones respiratorias a repetición (12). La evaluación funcional respiratoria puede ser orientadora como lo fue en este paciente y dependerá del grado de compromiso y localización de las lesiones; puede observarse OVAC intra $y / 0$ extratorácica (11-13). En nuestro caso la alteración funcional respiratoria observada coincidió con el examen clínico -sibilancias inspiratorias y espiratorias- con una curva flujo volumen que orientadora. No se conoce claramente la etiopatogenia de la traqueobroncopatía, aunque se ha descrito asociación a infección crónica por gram negativos, sinusitis y/o bronquitis crónica en situaciones de alteración del aclaramiento mucociliar, exposición a irritantes, alteraciones metabólicas o genéticas (10). En este paciente en que presenta solo compromiso del sistema respiratorio, se planteó el diagnóstico de una $F Q$ atípica o de una enfermedad relacionada al CFTR ya que solamente se pesquizó una mutación conocida para $F Q$ (F508del ) y además el tratamiento acorde al protocolo nacional de $F Q$ mostró una respuesta favorable (14).

\section{FIBROSIS QUISTTICA ATÍPICA}

Desde 1985 se conoce que el gen de la FQ está localizado en el brazo largo del cromosoma 7. En 1998 un panel de expertos estableció criterios diagnósticos basados en el fenotipo, en las mutaciones asociadas a $F Q$ y los rangos en exámenes diagnósticos tales como electrolitos en sudor y diferencia de potencial nasal (DPN) (1). La contribución del análisis molecular del gen que codifica para la proteína de CFTR ha permitido establecer correlación entre genotipo y fenotipo, habiéndose descrito hasta el momento cerca de 1.900 mutaciones (2). La frecuencia y los tipos de mutaciones varían en función de las razas y etnias. Los criterios genotípicos están primariamente basados en la identificación de 2 alteraciones en el gen de la CFTR y en general producen la pérdida completa de la función del canal, categorizadas en 6 distintas clases, siendo la que afecta la biosíntesis de la proteína las de clase I, su maduración las de clase II y su función las de clase III; estas se asocian a fenotipos severos. En las mutaciones de clase IV y V existe producción de la proteína que mantiene cierta actividad residual y están asociadas a suficiencia pancreática y enfermedad más leve $(1,2,4,7,8)$. La contribución de estas diferentes variantes alélicas del gen CFTR han sido evaluadas mediante estudios de correlación genotipo-fenotipo, con respecto a la variabilidad clínica 0 al grado de compromiso funcional $(15,16)$. La mutación clase IV resulta en una normal cantidad de CFTR, con menor función de ella en la membrana apical, disminuyendo así la conductancia del cloro. La mutación clase $\mathrm{V}$ está asociada con una disminución en la síntesis de CFTR activa resultando en una expresión disminuida en la membrana apical. La presencia de alelos poli T 5T y 7T sumado a una mutación de CFTR podrían influir en la expresión del CFTR, resultando en un fenotipo leve 0 no clásico $(2,16,17)$.

El TS mediante iontoforesis con pilocarpina es considerado el estándar de oro para confirmar el diagnóstico de $\mathrm{FQ}$. Valores sobre $60 \mathrm{mEq} / \mathrm{I}$ son consistentes con el diagnóstico de $F Q$, bajo $30 \mathrm{mEq} / \mathrm{l}$ en lactantes menores de 6 meses 0 bajo $40 \mathrm{mEq} / \mathrm{l}$ en niños mayores se considera negativo $(1,5)$. Mediciones entre 40 y $60 \mathrm{mEq} / \mathrm{l}$ se han asociado a FQ atípica, lo que requiere de mayor evaluación ya sea con la diferencia de potencial nasal -que ha demostrado ser muy útil en estos casos- el estudio genético y la correlación con el cuadro clínico $(2,5,21)$. Hoy se sabe que un TS normal no excluye en forma definitiva la enfermedad; un ejemplo de ello podría ser el caso descrito. La mayoría de los pacientes en Europa y Estados Unidos han sido evaluados genéticamente; por ejemplo en Inglaterra y Francia cerca de un 95\% de ellos han sido genotipificados $(2,3)$. Con el advenimiento de las terapias personalizadas que tratan el defecto del CFTR se hace necesario su evaluación integral. El estudio genético es aún muy laborioso y de alto costo, motivo por el cual se utiliza en general en pacientes con alta sospecha clínica de FQ, en los cuales el TS es normal y el análisis de mutaciones frecuentes no puede confirmar la enfermedad. Esto en ocasiones identifica alteraciones cuyas consecuencias patológicas no son tan claras, ya sea polimorfismos, mutaciones leves que causan enfermedad relacionada al CFTR o mutaciones causantes de FQ propiamente tal (21)

\section{DIFERENCIA DE POTENCIAL NASAL (DPN)}

Dado que en estos casos no siempre se dispone del estudio genético ampliado para las casi 2000 mutaciones 
descritas, se desarrolló la DPN, método diagnóstico que es más sensible y específico. Este examen es especialmente útil en pacientes con concentración de cloro en sudor normal y en los que no se identifican las 2 mutaciones del gen de la $F Q$; consiste en la demostración in vivo del transporte iónico anormal por alteración de la función de la proteína a través de algún epitelio del organismo (22). Esta diferencia de potencial es negativa respecto de la submucosa, ya que en todos los epitelios respiratorios es isoeléctrica, al igual que el tejido celular subcutáneo. Para la realización de la DPN se requieren 2 electrodos conectados a un voltímetro, uno colocado sobre la mucosa nasal del cornete inferior, y otro en el tejido celular subcutáneo del antebrazo. Un valor inferior a $-40 \mathrm{mV}$ se considera patológico. En sujetos sanos los valores obtenidos no sobrepasan nunca este valor. Se requieren 2 determinaciones anormales de DPN registradas en 2 días separados para aceptar la disfunción de la CFTR (22). Con este conocimiento y gracias a esta prueba, el índice de sospecha de FQ ha aumentado, todo esto ha sido acompañado del mayor desarrollo de los estudios genéticos, lo que ha permitido detectar un número creciente de mutaciones nuevas del gen $F Q$, pero que no se habían asociado a enfermedad. Este hecho ha cambiado el concepto de la FQ, que hoy obliga a considerar a esta enfermedad con un espectro clínico más amplio y ha permitido aumentar en forma creciente el diagnóstico en pacientes adultos (20-23).

\section{ENFERMEDAD RELACIONADA AL CFTR}

Los individuos que muestran fenotipo de $F Q$ con un órgano o sistema afectado, un TS normal o poco elevado, son descritos como portadores de enfermedad no clásica 0 atípica (21). Generalmente estos pacientes tienen suficiencia pancreática con enfermedad pulmonar leve con 2 mutaciones del CFTR en que al menos una resulta en expresión o función parcial. En la actualidad esta situación se denomina enfermedad relacionada al CFTR, siendo ejemplos de ellas la ausencia congénita de conductos deferentes, la pancreatitis crónica idiopática, las bronquiectasias aisladas diagnosticadas a edades mayores, la cirrosis biliar con hipertensión portal y los pólipos nasales, entre otras (15-19,21,23-25.) La enfermedad pulmonar puede ser menos severa que en la FQ clásica, pero puede mostrar similitudes fenotípicas como infección crónica predominante en lóbulos superiores por Pseudomona aeruginosa (16). Algunos estudios han encontrado mutaciones en bronquiectasias idiopáticas, pero no cumplen los criterios diagnósticos de $F Q$, debido a que no en todos los casos fue posible identificar las dos mutaciones. En estos pacientes las causas de las bronquiectasias podrían ser de origen multifactorial $(18,23)$, por esto la enfermedad pulmonar leve, con bronquiectasias idiopáticas en pacientes mayores exige un estudio de FQ atípica con TS, DPN y estudio genético, especialmente para mutaciones leves $(19,23)$. La pancreatitis recurrente idiopática se ha asociado en un 10 a $20 \%$ a la portación de dos genes CFTR anormales, en que uno está clasificado como leve (24). Aunque la pancreatitis en infrecuente en individuos con FQ clásica, su incidencia es mayor en aquellos casos que presentan suficiencia pancreática $(15,25)$. Otro ejemplo de un único órgano involucrado es la infertilidad masculina por ausencia de vas deferens, lo que ocurre con una frecuencia de 1 a $2 \%$ de los hombres infértiles; en estos casos la mutación del CFTR está presente en cerca de 50-70\% de los afectados, siendo las más comunes las F508 del/R117H (3). El genotipo típicamente incluye una mutación menos severa y resulta en un $10 \%$ de funcionalidad residual de la proteína CFTR lo cual explica el espectro de estos fenotipos menos severos (8). Las mutaciones son variantes intermedias entre clase IV y clase V. Una significativa proporción de estos hombres afectados tiene sinusitis crónica y una diferencia de potencial nasal alterada $(8,15)$

\section{SÍNDROME METABÓLICO ASOCIADO AL CFTR}

Se han clasificado como portadores del llamado síndrome metabólico asociado al CFTR a niños asintomáticos pesquisados como posible $F Q$ en el tamizaje neonatal en los cuales no puede confirmarse o excluirse la enfermedad por presentar prueba de sudor indeterminada y menos de 2 alelos mutados causantes de FQ. Esto incluye a los casos en los cuales la estrategia involucra la determinación de ADN y se pesquisan 2 mutaciones, siendo una de ellas no conocida como causante de la enfermedad (26) Estos niños deben ser seguidos en un centro especializado, porque que se ha descrito que ya desde los 3 años podrían presentar algunos síntomas de la enfermedad $(26,27)$.

\section{CONCLUSION}

Los fenotipos menos severos de $F Q$ tienen poco compromiso multisistémico y las mutaciones del CFTR involucradas son menos comunes. Cuando esto ocurre, especialmente en adolescentes mayores o adultos, puede ser indicativo de $F Q$ atípica, pero hacer el diagnóstico de ello no es fácil.

\section{Los autores declaran no tener conflictos de interés}

\section{REFERENCIAS}

1. Rosentain BJ. The diagnosis of cystic fibrosis: a consensus statement. Cystic Fibrosis Foundation Panel. J Pediatr 1998;132: 589-595

2. Mogayzel P, Flume P, Update in Cystic Fibrosis 2009. Am J Respir Crit Care Med 2010; 181: 539-544

3. Zielenski J. Genotype and phenotype in CF. Respir 2000 ; 67: 117-133

4. Fielbaum 0. Avances en fibrosis quística. Rev Med Clin Condes 2011; 22(2) 150-159

5. Borowitz D, Robinson KA, Rosenfeld M, Davis SD, Sabadosa KA, Spear SL, Michel SH, Parad RB, White TB, Farrell PM, Marshall BC, Accurso FJ. Cystic Fibrosis Foundation. Evidence-Based Guidelines for Management of Infants with Cystic Fibrosis. J Pediatr 2009;155:S73-93

6. Smyth AR, Bell SC, Bojcin S, Bryon M, Duff A, Flume P, 
Kashirskaya N, Munck A, Ratjen F, Schwarzenberg SJ, Sermet-Gaudelus I, Southern KW, Taccetti G, Ullrich G, Wolfe S; European Cystic Fibrosis Society. Standards of Care: Best Practice guidelines. J Cyst Fibross 2014; 13:S23 - S42

7. Boyle MP. Nonclassic cystic fibrosis and CFTR-related diseases. Curr Opin Pulm Med 2003 ; 9:498-503

8. Kerem E. Atypical CF and CF related diseases. Paediatr Respir Rev 2006;7(Suppl 1):S144-6

9. Celedón C, de Grazia DA. Traqueobroncopatía osteocondroplástica: reporte de un caso y revisión de la literatura. Rev Otorrinolaringol Cir Cabeza Cuello 2010; 70: 147-152

10. Xing $X Q$, Liu $Y H$, Li $Y L$, Zhang HY, Zhang $Y X$, Wu XW. Tracheobronchopathia osteochondroplastica: imaging and bronchoscopic findings. Ann Thorac Surg. 2015;99:e97

11. Nielsen SW, Stevens JR, Dion GR, Howard NS. Dyspnea, Dysphonia, and Cough. Varied Presentations of Tracheobronchopathia Osteochondroplastica. Ann Otol Rhinol Laryngol. $2015 ; 124: 829-33$

12. Luo S, Wu L, Zhou J, Xu S, Yang Q, Li Y, Shen H, Zhang S. Tracheobronchopathia osteochondroplastica: two cases and a review of the literature. Int $\mathrm{J}$ Clin Exp Pathol 2015;8:8585-90

13. Sun J, Xie L, Su X, Zhang X. Tracheobronchopathia osteochondroplastica: Case report and literature review. Respir Med Case Rep. 2015;26;15:14-7

14. MINISTERIO DE SALUD, CHILE. Guía Clínica Fibrosis Quística Santiago: Minsal, 2012

15. Paranjape SM, Zeitlin PL. Atypical cystic fibrosis and CFTRrelated diseases. Clin Rev Allergy Immunol 2008;35:11623

16. Dal'Maso VB, Mallmann L, Siebert M, Simon L, SaraivaPereira ML, Dalcin P de TR. Diagnostic contribution of molecular analysis of the cystic fibrosis transmembrane conductance regulator gene in patients suspected of having mild or atypical cystic fibrosis. J Bras Pneumol 2013;39:181-9

17. Schram CA. Atypical cystic fibrosis. Identification in the primary care setting Can Fam Physician 2012;58:1341-5

18. Van Biezen P, Overbeek SE, Hilvering C. Cystic fibrosis in a 70 year old woman. Thorax 1992;47:202-3

19. Gan KH, Geus WP, Bakker W, Lamers CB, Heijerman HG. Genetic and clinical features of patients with cystic fibrosis diagnosed after the age of 16. Thorax 1995;50:1301-4

20. Mainz J, Hammer U, Rokahr C, Hubler A, Zintl F, Ballmann M. Cystic fibrosis in 65 and 67 year-old siblings. Clinical feature and nasal potential difference measurement in patients with genotypes F508del and $2789+5 \mathrm{G} \rightarrow \mathrm{A}$. Respiration 2006;73:698-704

21. Southern KW. Cystic fibrosis and formes frustes of CFTRrelated disease. Respir 2007; 74:241-51

22. Domingo-Ribas $\mathrm{C}$, Bosque-García M. Prueba de la diferencia de potencial nasal para el diagnóstico de fibrosis quística. Arch Bronconeumol. 2006;42:33-8

23. Luisetti M. Genetics of idiopathic disseminated bronchiectasis. Semin Respir Crit Care Med 2003; 24: 179-184

24. Cohn JA. Relation beetween mutations of the CF gene and idiopathic pancreatitis. NEJM 1998; 339: 653-61158

25. Sultan M, Werlin S, Venkatasubramani N. Genetic prevalence and characteristics in children with recurrent pancretaitis. J Pediatr Gastroenterol Nutr. 2012; 54:645-50

26. Nelson M, Adamski C, Tluczek A. Clinical practices for intermediate sweat test following abnormal cystic fibrosis newborn screens. J Cystic Fibros 2011;10:460-65

27. Prach L, Koepke R, Kharrazi M, Keiles S, Salinas D, Reyes $\mathrm{MC}$ et al. Novel CFTR Variants Identified during the First 3 Years of Cystic Fibrosis Newborn Screening in California. J Mol Diagn 2013, 15: 710e722 